\title{
Baseline and stress-induced corticosterone levels are heritable and genetically correlated in a barn owl population
}

\author{
Paul Béziers $\mathbb{1}^{1,2} \cdot$ Luis M. San-Jose $^{1} \cdot$ Bettina Almasi $^{2} \cdot$ Lukas Jenni $^{2} \cdot$ Alexandre Roulin $^{1}$
}

Received: 26 November 2018 / Revised: 25 January 2019 / Accepted: 8 February 2019 / Published online: 5 March 2019

(c) The Genetics Society 2019

\begin{abstract}
The hypothalamic-pituitary-adrenal (HPA) axis is responsible for the regulation of corticosterone, a hormone that is essential in the mediation of energy allocation and physiological stress. As a continuous source of challenge and stress for organisms, the environment has promoted the evolution of physiological adaptations and led to a great variation in corticosterone profiles within or among individuals, populations and species. In order to evolve via natural selection, corticosterone levels do not only depend on the strength of selection exerted on them, but also on the extent to which the regulation of corticosterone is heritable. Nevertheless, the heritability of corticosterone profiles in wild populations is still poorly understood. In this study, we estimated the heritability of baseline and stress-induced corticosterone levels in barn owl (Tyto alba) nestlings from 8 years of data, using a multivariate animal model based on a behavioural pedigree. We found that baseline and stress-induced corticosterone levels are strongly genetically correlated $(r=0.68-0.80)$ and that the heritability of stress-induced corticosterone levels $\left(h^{2}=0.24-0.33\right)$ was moderate and similar to the heritability of baseline corticosterone levels $\left(h^{2}=0.19-0.30\right)$. These findings suggest that the regulation of stress-induced corticosterone and baseline levels evolves at a similar pace when selection acts with the same intensity on both traits and that contrary to previous studies, the evolution of baseline and stress-induced level is interdependent in barn owls, as they may be strongly genetically correlated.
\end{abstract}

\section{Introduction}

There is a lack of knowledge about the potential of the endocrine system to respond to selection, compared with morphological or life-history traits (Charmantier and Garant 2005; Kempenaers et al. 2008; Pavitt et al. 2014; Zera et al. 2007). Yet, hormones such as glucocorticoids play an important role in regulating and coordinating physiological and behavioural functions in order to adaptively respond to internal demands and external challenges (Breuner et al. 2008;

These authors contributed equally: Lukas Jenni, Alexandre Roulin

Supplementary information The online version of this article (https:// doi.org/10.1038/s41437-019-0203-5) contains supplementary material, which is available to authorised users.

Paul Béziers

paul.beziers@unil.ch

1 Department of Ecology and Evolution, University of Lausanne, Lausanne, Switzerland

2 Swiss Ornithological Institute, Sempach, Switzerland
Romero 2004; Wingfield et al. 1998). Moreover, hormones typically affect the expression of multiple traits, which may induce constraints and trade-offs in the evolution of lifehistory traits (Flatt et al. 2005; Zera et al. 2007). Despite the acknowledged importance of hormones in selective processes, little is known about the contribution of genetics to variation in glucocorticoid levels, and consequently, about the potential of the hypothalamic-pituitary-adrenal (HPA) axis in regulating them to respond to selection in natural conditions. Determining the heritable variation underlying the secretion of glucocorticoids is therefore required to predict better how populations might adaptively respond to perturbations in their habitat, including global change, urbanisation or other types of human disturbance.

The HPA axis regulates the secretion of glucocorticoids (corticosterone in avian and non-avian reptiles and cortisol in most mammals and fish) in the bloodstream from where they are distributed into the tissues and organs in order to regulate physiological processes. At baseline levels, glucocorticoids play an important role in daily life processes, including energy intake (Dallman et al. 1993; King 1988), water/salt balance (Bramanti et al. 1997) and glucose supply 
in cells (Kuo et al. 2015). They also support the allocation of metabolic demands between life-history traits and between life-history stages (Crespi et al. 2013; Landys et al. 2006; Sapolsky et al. 2000). Thus, glucocorticoid baseline levels are strongly influenced by environmental factors (Romero and Wingfield 2016), maternal effects and early life experiences, which can all have long-term effects on the secretion of corticosterone by the HPA axis (Haussmann et al. 2012; Henriksen et al. 2011). Further increase in glucocorticoid levels can be triggered by unpredictable stressful events leading to the so-called "stress-induced glucocorticoid response", which allows animals to adjust their physiology and behaviour to, for instance, inclement weather conditions, predator attacks or food shortage (Landys et al. 2006; Rodrigues et al. 2009; Sapolsky et al. 2000). Both baseline and stress-induced glucocorticoid levels have been shown to be associated with reproductive success and survival in different taxa, indicating that the HPA axis might often be under selection (Bonier et al. 2009a; Breuner et al. 2008, but see Bonier and Martin 2016 for discussion). In some scenarios, such as in urban environments, individuals exhibit shifts in the response to the HPA axis that generally seem to promote adaptation to environmental conditions (Abolins-Abols et al. 2016; Atwell et al. 2012; Fokidis et al. 2009; Müller et al. 2007). This suggests that glucocorticoid levels respond to selection, but the limited understanding of heritable variation in corticosterone levels in wild populations impedes to discriminate if those differences are due to either plastic responses (Bonier and Martin 2016), or to evolved genetically based differences.

Determining to what extent the HPA axis regulation of glucocorticoids is genetically determined is necessary for a better understanding of the proximate mechanisms underlying variation in glucocorticoid levels, the evolutionary consequences of such variation and the direct and indirect responses of the HPA axis to processes promoting its adaptive evolution (Bonier and Martin 2016; Cox et al. 2016; Hau and Goymann 2015). There is evidence from studies made in captivity that glucocorticoid levels respond to artificial selection, and hence that the HPA axis can potentially evolve under selection. These studies showed that the heritability, $h^{2}$, varies between 0.23 and 0.70 for baseline (Fairbanks et al. 2011; Fevolden et al. 1999; Kadarmideen and Janss 2007) and 0.04 and 0.60 for stressinduced glucocorticoid levels (Brown and Nestor 1973; Cox et al. 2016; Evans et al. 2006; Odeh et al. 2003; Pottinger and Carrick 1999; Satterlee and Johnson 1988). These values are comparable to heritability for other physiological traits (Coviello et al. 2010; Mills et al. 2012; Pavitt et al. 2014; Travison et al. 2014) but tend to be lower than heritability for morphological traits (Mousseau and Roff 1987). Unfortunately, little is known about the situation prevailing in wild populations where natural selection comes into play. Moreover, heritability is a measure of the relative contribution of genetic vs. environmental variation to the phenotypic variation of a trait for a given population and at a given time point (i.e., heritability is a population-level measure that may differ between populations and cannot be extrapolated to other populations). Thus, estimates obtained in laboratory environments might not properly reflect the levels of heritability in wild outbred populations, particularly for glucocorticoids, which are important transducers between an organism and its environment. In natural conditions, the environment is more variable and population structure is less homogeneous than in laboratory conditions where there is a smaller contribution of the environment to the variation in an individual phenotype, generally resulting in higher heritability estimates (Charmantier and Garant 2005; Huey and Rosenzweig 2009; Sgro and Hoffmann 2004).

To understand how baseline and stress-induced glucocorticoid levels evolve, it is also important to infer if both traits are genetically correlated and thereby able to evolve independently. Although the effects of baseline and stressinduced levels are mediated by different protein receptors and may function as two complementary hormonal systems (Landys et al. 2006; Romero 2004), both baseline and stress-induced glucocorticoid levels depend on certain shared pathways that allow them to evolve jointly to some extent. Both are regulated by the secretion of corticotropinreleasing hormone $(\mathrm{CRH})$, which activates the HPA axis by stimulating the pituitary secretion of adrenocorticotropic hormone (ACTH) into the bloodstream, from where it flows to the adrenal glands and induces the secretion of glucocorticoids. The secretion of CRH is influenced by many factors, such as the circadian rhythm (Buckley and Schatzberg 2005), physical activity (Chennaoui et al. 2002; Kawashima et al. 2004), blood glucocorticoid levels and stressful events (Carsia and Harvey 2000; Harvey and Hall 1990). There is evidence that baseline and stress-induced levels may evolve indirectly in response to selection on behavioural traits (Albert et al. 2008; Carere et al. 2003; Garland et al. 2016; Stowe et al. 2010), suggesting that both traits are genetically correlated. For instance, Albert et al. (2008) observed lower levels of both baseline and stressinduced glucocorticoid levels in rats selected for tameness, compared with those selected for aggressiveness. However, selection on personality traits does not always cause a correlated change in both baseline and stress-induced glucocorticoid levels (Baugh et al. 2012). Similarly, selection for high and low stress-induced glucocorticoid levels did not induce a correlated change in baseline glucocorticoid levels neither in the rainbow trout (Oncorhynchus mykiss) (Pottinger and Carrick 1999) nor in the Japanese quail (Coturnix coturnix japonica) (Cockrem et al. 2010). 
Moreover, the two studies that have directly tested for the existence of a genetic correlation between these traits in wild populations did not find evidence for genetic covariation in baseline and stress-induced response (Jenkins et al. 2014; Stedman et al. 2017). Altogether, these results suggest that the genetic covariation between baseline and stress-induced glucocorticoid levels might not be a consistent pattern across all populations and species, evidencing that more data from different taxa are still needed.

Over a period of 8 years, we measured baseline and stress-induced corticosterone levels in 1,211 nestling barn owls in order to estimate the heritability of these two components of the HPA axis. We performed animal models using a pedigree established with a data set comprising a 21year monitoring period, including a total of 8,596 individuals. To the best of our knowledge, only two studies (Jenkins et al. 2014; Stedman et al. 2017) have investigated the heritability of baseline (American barn swallows, Hirundo rustica: $h^{2}=0.15$; tree swallows, Tachycineta bicolour: $\left.h^{2}=0.13\right)$ and stress-response corticosterone levels in the wild (American barn swallows: $h^{2}=0.34$; tree swallows: $\left.h^{2}=0.18\right)$. We also investigated the extent to which baseline and stress-induced response levels are genetically correlated.

\section{Methods}

\section{Data collection}

Our study was performed with a population of barn owls breeding in nest boxes fixed to barns in western Switzerland $\left(46^{\circ} 49^{\prime} \mathrm{N}, 06^{\circ} 56^{\prime} \mathrm{E}\right)$ and that has been monitored since 1992. This nocturnal species lives in open landscapes and preys mainly upon small mammals. In our study site, 2-12 eggs are laid from mid-February to the beginning of August (Béziers and Roulin 2016). Eggs hatch asynchronously because they are laid every 2-3 days and incubation starts immediately after the first egg is laid. This generates a pronounced age/size hierarchy among the progeny. The first flight occurs at ca. 55 days of age. We ringed all nestlings and most breeding adults.

Over an extended period of time (8 years: 2004-2006, 2009-2012 and 2016), we collected blood samples from 570 male and 641 female nestlings (Table S1) (molecular sex identification following (Py et al. 2006)) born in 307 different nests (mean of 3.94 nestlings per nest, 1.55 SD). Some of the nestlings used in this study were part of other experimental manipulations (Almasi et al. 2009; Almasi et al. 2012; Müller et al. 2009; Roulin et al. 2008). To avoid any biases due to these experimental manipulations, we only considered samples that were taken before the manipulations. For some nestlings, pre-experimental samples were taken at a very young age $(<15$ days old); however, samples taken on individuals at older ages could also be included in this study because they were part of control or treatment groups previously shown to have no effect on corticosterone levels.

The mean age of nestlings for which we had baseline measurements and for which we had stress-induced corticosterone measurements (see the Assessment of baseline and stress-induced corticosterone levels section) was 37.9 days $( \pm 11.2 \mathrm{SD}$, range: $6-67)$ and 38.2 days ( \pm 11.1 SD, range: 6-67), respectively. In some species, the stress-induced corticosterone response of young individuals varies strongly with age or hardly exists before fledging. In our population, nestlings showed the capacity to mount corticosterone levels in response to a stressor, already at an early age ( 6 days, unpublished data). We did find a significant relationship between age and corticosterone levels (Fig. S1, although age accounted for less than 3\% and $4 \%$ of the variation in baseline and stress-induced corticosterone stress-response levels, respectively). These findings indicate that variation in age in our sampled population has little incidence on our results (see also Table S2).

Eggs (occasionally nestlings, 110 over 1,075 eggs) were cross-fostered between pairs of nests that had similar laying dates (mean difference in laying date between pairs of cross-fostered nests ranged between 0 and 17 days with a mean of 1.7 days $\pm 2.4 \mathrm{SD}$ ). When the clutch size between a pair of nests was of similar size $( \pm 1 \mathrm{egg})$, we swapped all eggs. When there was a difference of more than one egg or the laying date between the pair of nests available for crossfostering was marked (ca. 17 days), we either cross-fostered the same number of eggs between pairs of randomly chosen nests or swapped the oldest nestlings from one nest with the youngest of the other nest. In the latter case, we made sure to keep intact the within-brood age hierarchy by swapping eggs at the same stage of incubation. Of the 1,112 nestlings for which we have baseline corticosterone levels, 697 (62.7\%) were cross-fostered and $730(66.0 \%)$ out of the 1,106 nestlings for which we have stress-induced levels were cross-fostered. In most cases, individuals were crossfostered before hatching (594 [85.2\%] for individuals with baseline measurements, and 642 [87.9\%] for individuals with stress-induced measurements). On average, eggs were cross-fostered ca. 10 days before they hatched (9.7 \pm 5.9 SD). Individuals that were cross-fostered after hatching were on average 3.7 days old $( \pm 2.6 \mathrm{SD})$.

\section{Assessment of baseline and stress-induced corticosterone levels}

We measured baseline and stress-induced corticosterone levels in a total of 1,112 (522 males, 590 females from 302 distinct nests) and 1,106 (519 males, 587 females from 282 different nests) nestlings, respectively. For 1,007 nestlings 
(471 males, 536 females), we had both baseline and stressinduced corticosterone levels. Nestlings were sampled for blood between $7.33 \mathrm{~h}$ in the morning and $20.42 \mathrm{~h}$ in the evening. Baseline samples were taken within $3^{\prime}\left(1^{\prime} 58^{\prime \prime} \pm\right.$ 39 " average sampling time and SD) after opening the nest box (Romero and Reed 2005). Although within $3 \mathrm{~min}$ the effect of sampling time was significant $\left(t_{1,110}=6.67, P<\right.$ $0.0001)$, it only explained a small portion of the variance in baseline corticosterone levels $\left(R^{2}=0.04\right)$. Once the first blood sample was taken, the individuals were weighed to the nearest $g$ and their wing and tarsus measured to the nearest $\mathrm{mm}$. Nestlings were then individually kept in cloth bags until a second blood sample was taken within $19^{\prime}$ to $32^{\prime} 45^{\prime \prime}$ (mean: $24^{\prime} 18^{\prime \prime} \pm 2^{\prime} 42^{\prime \prime}$ SD) to measure stressinduced corticosterone levels.

The blood samples were collected with heparinised capillary tubes, immediately centrifuged and the plasma was stored in liquid nitrogen separately from the red blood cells. Within less than $24 \mathrm{~h}$, the samples were stored at $-20^{\circ} \mathrm{C}$ until further analysis. Plasma corticosterone levels were determined using an enzyme immunoassay (see for details Müller et al. 2006). Briefly, $10 \mu \mathrm{L}$ of plasma was added to $190 \mu \mathrm{L}$ of water, and from this solution, we extracted corticosterone with $4 \mathrm{~mL}$ of dichloromethane, which was re-dissolved in phosphate buffer and measured in triplicate in the enzyme immunoassay. The dilution of the corticosterone antibody (Chemicon; crossreactivity: 11-dehydrocorticosterone $0.35 \%$, progesterone $0.004 \%$, 18-OH-DOC $0.01 \%$ cortisol $0.12 \%$, 28-OH-B $0.02 \%$ and aldosterone $0.06 \%$ ) was 1:8,000. We used HRP $(1: 400,000)$ linked to corticosterone as the enzyme label and ABTS as a substrate. The concentration of corticosterone in plasma samples was calculated by using a standard curve run in duplicate on each plate. If the corticosterone concentration was below the detection threshold of $1 \mathrm{ng} \mathrm{mL}^{-1}$, the analysis was repeated with 15 or $20 \mu \mathrm{L}$ of plasma. If the concentration was still below the detection limit, we assigned to the sample the lowest detectable concentration value $\left(1 \mathrm{ng} \mathrm{mL}^{-1}\right)$. Plasma pools from chicken with a low and high corticosterone concentration were included as internal controls on each plate. Intra-assay variation ranges from 3 to $20 \%$ and inter-assay variation from 7 to $25 \%$, depending on the concentration of the internal control and the year of analysis. Baseline corticosterone levels ranged between 0.49 and $58.3 \mathrm{ng} \mathrm{mL}^{-1}$ (mean $\pm \mathrm{SD}: 7.3 \pm 7.1$ ) and stress-induced corticosterone levels between 5.1 and $147.6(60.1 \pm 24.6)$. Stress-induced corticosterone levels were significantly correlated with baseline corticosterone (Pearson's correlation $r=0.36,95 \% \mathrm{CI}$ : $0.31-0.42, t_{1,005}=12.41, P<0.001$, Fig. S2).

\section{Statistics}

We used an "animal model" approach to estimate the heritability of baseline and stress-induced corticosterone levels in nestling barn owls (Wilson et al. 2010). The animal model is a linear mixed model that estimates the additive genetic variance of a trait of interest from a population's pedigree. The pedigree is used to control for relatedness when estimating the breeding value of an individual (i.e., the additive genetic effect on a phenotype).

\section{Pedigree}

We used a wild social pedigree (Pemberton 2008): a pedigree built using paternities assessed from field behavioural observations of male and female parental care (i.e., the adults observed incubating and/or feeding the nestlings were assigned as parents; see also San-Jose et al. 2017). Because the rate of extra-pair paternity is very low in our population ( $\sim 1 \%$, Ducret et al. 2016; Henry et al. 2013), mis-assigned paternity links based on behavioural observations are expected to be negligible and have a small incidence on the models' estimates (Charmantier and Reale 2005). The full pedigree consisted of 8,815 individuals captured as breeders or nestlings between 1994 and 2016. Excluding the pedigree "founders" (1,202 adults of an unknown nest of origin), only $2.89 \%$ of the individuals in the pedigree have an unknown mother and $10.35 \%$ of the individuals, an unknown father (1.2\% belong to the same brood with both parents unknown). A unique "dummy" mother and/or father was assigned to each one of these broods. The pedigree pruned for individuals, for which baseline corticosterone levels were measured, was composed of 1,601 individuals (including 375 founders) with 1,226 maternity and paternity links, 2,819 full sibs (4,382 maternal sibs, 4,899 paternal sibs), 1,563 maternal half-sibs, 2,080 paternal half-sibs, 295 maternal grandmothers and grandfathers and 479 paternal grandmothers and grandfathers. The pedigree pruned for individuals with known stress-induced levels was composed of 1,576 individuals (including 364 founders) with 1,212 maternity and paternity links, 3,043 full sibs (4,615 maternal sibs, 5,056 paternal sibs), 1,572 maternal half-sibs, 2,013 paternal half-sibs, 315 maternal grandmothers and grandfathers and 459 paternal grandmothers and grandfathers. Both pedigrees had a maximum pedigree depth of six generations, which according to Balloux et al. (2004) is expected to yield accurate estimates of relatedness among the individuals in the pedigree. The pedigree depth and sample size are larger than that in previous studies testing for genetic (co)variation in baseline and stress-induced corticosterone levels in wild animal populations (Jenkins et al. 2014; Stedman et al. 2017).

\section{Animal models}

The animal models were fitted using the MCMCglmm function implemented in the $M C M C g l m m \mathrm{R}$ package 
(Hadfield 2010). Baseline and stress-induced corticosterone levels were considered together in the same multivariate analyses, which allowed us to estimate the heritability of each trait as well as their genetic correlation. In an initial full model, the inverse matrix of relatedness among individuals, as estimated from the pedigree, was included together with the year and brood identity as random effects, while no fixed effects were included. The variance estimated from the pedigree represents the additive genetic variance $\left(V_{\mathrm{A}}\right)$, the variance associated with brood identity represents the rearing environmental variance $\left(V_{\mathrm{E}}\right)$ and the variance associated with year represents the yearly variance $\left(V_{\mathrm{Y}}\right)$. From these variance components and the residual variance $\left(V_{\mathrm{R}}\right)$, we calculated narrow-sense heritability $\left(h^{2}\right)$ as $V_{\mathrm{A}} / V_{\mathrm{P}}\left(V_{\mathrm{P}}=\right.$ total phenotypic variance). Within the same model, we modelled the variance terms of the random effects separately for baseline and stress-induced corticosterone levels (option idh in MCMCglmm; Hadfield 2010), except for the genetic additive variance, which was modelled separately for baseline and stress-induced corticosterone levels but also taking into consideration their covariation (i.e., genetic covariance, option $u s$ in MCMCglmm).

To test whether modelling additive genetic variance and covariance in corticosterone levels improved model fit, we compared the full model described above with a second model, considering additive genetic variance terms per trait and zero genetic covariance between the traits, and a third model considering no additive genetic variance for any of the traits. Models were compared, based on their DIC (deviance information criterion) values. All models were run in duplicate to estimate the variation in DIC values owing to model mixing. The differences between duplicates were all small $(\Delta \mathrm{DIC} \leq 1.1)$.

We repeated all the models presented above, including the fixed effects of different covariates that may affect corticosterone levels in the barn owl (Almasi et al. 2015; Almasi et al. 2010; Romero and Reed 2005; Roulin et al. 2010). Including fixed effects into animal models must be carefully considered, as it may yield downward estimates of residual variance and thereby inflated estimates of heritability (see Wilson 2008 for a detailed discussion on the topic). Nonetheless, modelling fixed effects allows us to assess to what extent our heritability estimates could be biased by nonbiological sources of variance that were inherent to the sampling procedure (e.g., sampling time) or to the encountered sampling population (e.g., age of the individuals). We included the following covariates: sampling time (i.e., duration between the moment when nestlings were first disturbed and blood sampling), sampling date (i.e., Julian date), sampling time of the day (i.e., hour of capture), sex, nestling age, rank in the brood, brood size and condition (i.e., residuals from the relation between mass and wing length of nestlings). Covariates were considered in interaction with trait (a categorical variable reflecting whether a corticosterone measurement in the model corresponded to baseline or stress-induced levels). This is required given that: i. the same covariate (e.g., age) may associate with baseline differently than with stress-induced levels, or ii. the value of the covariate changes with trait: i.e., from baseline to stress-induced measurements (e.g., sampling time). To further test the robustness of estimates of the animal models, we ran the animal models considering only those individuals that were cross-fostered or considering a smaller range of ages. These models gave qualitatively similar results than those presented in the Results section (see Table S3 and Fig. S3).

All models were fitted with inverse Wishart priors, as described by Hadfield (2010), and ran for 10,300,000 iterations with a burning phase of 300,000 iterations and a sampling interval of 2,000 iterations. The mean effective sample size for all model parameters was 4,730 and the lowest effective sample size was 3,769. To assess the models, we visually inspected the convergence of the chains and checked the congruence of the posterior distributions of the different parameters between runs. Baseline levels were log-transformed and a power of 0.7 transformation to stressinduced levels was used to improve the normality of the data.

\section{Results}

The model considering a genetic additive variance term for baseline and stress-induced corticosterone levels and their genetic covariation was found to fit the data better than the models considering no genetic covariation (the second best model) or no additive genetic variance for both traits (the third best model, Table 1$)$. The heritability estimate $\left(h^{2}\right)$ of the best model for baseline corticosterone levels $\left(h^{2}=0.303\right.$, 95\% Bayesian credible interval, BCrI: 0.163-0.396, $N=$ 1,112) was similar to the heritability estimate for stressinduced levels $\left(h^{2}=0.326,95 \%\right.$ BCrI: $0.194-0.471, N=$ 1,106; Fig. 1, Table 2). The genetic correlation between baseline and stress-induced corticosterone levels was high ( $r$ $=0.803$, 95\% BCrI: 0.685-0.895). The rearing environment (brood ID) explained a small part of the variance (10\%) in baseline corticosterone levels (Fig. 1, Table 2), whereas the part of variance explained by year was higher (23\%) albeit with a larger $95 \%$ BCrI. In stress-induced response levels, both the year and the rearing environment explained a small part of the variance $(<11 \%)$ (Fig. 1, Table 2). A large part of the variance for baseline $(33 \%)$ and stress-induced levels $(42 \%)$ remained unexplained (Fig. 1, Table 2).

When including the covariates (date, hour, sampling time, age and sex of nestlings) into the models, the model 
considering a genetic additive variance term for baseline and stress-induced corticosterone levels and their genetic covariation was also found to fit the data better than the other models tested (Table 1, Table S2). This model showed

Table 1 Comparison of animal models including different additive genetic variation and covariation terms for baseline and stress-induced corticosterone levels in nestling barn owls

\begin{tabular}{|c|c|c|c|c|}
\hline & \multicolumn{2}{|l|}{ Genetic } & \multirow[t]{2}{*}{ DIC } & \multirow[t]{2}{*}{$\Delta \mathrm{DIC}$} \\
\hline & Variance & Covariance & & \\
\hline \multicolumn{5}{|c|}{ Models without covariates } \\
\hline Model 1 & Yes & Yes & $8,407.4$ & 0 \\
\hline Model 2 & Yes & No & $8,661.8$ & 254.4 \\
\hline Model 3 & No & No & $8,743.0$ & 335.6 \\
\hline \multicolumn{5}{|c|}{ Models including covariates } \\
\hline Model 1 & Yes & Yes & $8,144.5$ & 0 \\
\hline Model 2 & Yes & No & $8,272.4$ & 127.9 \\
\hline Model 3 & No & No & $8,367.7$ & 223.2 \\
\hline
\end{tabular}

Note: In model 1 , we estimated the additive genetic variances for baseline and stress-induced corticosterone levels and their genetic covariation. In model 2 , we estimated the additive genetic variances for baseline and stress-induced corticosterone levels but considered that genetic covariance equals zero. Finally, in model 3, we considered no additive genetic (co)variance for corticosterone levels. In each model, we added the identity of the brood and year as random factors. Shown are the DIC (deviation information criteria) values for each model and the differences of each model to the model with the lowest DIC ( $\triangle$ DIC) for models without or with covariates included somewhat similar estimates as the model without covariates (Table 2, Fig. 1), although the estimates of additive genetic variance for baseline $\left(h^{2}=0.189,95 \%\right.$ BCrI: 0.104-0.306) and stress-induced response levels $\left(h^{2}=0.241,95 \% \mathrm{BCrI}\right.$ : 0.116-0.395), as well as, their level of genetic correlation were lower ( $r=0.683,95 \%$ BCrI: $0.051-0.820)$ than in the model without covariates.

\section{Discussion}

The evolutionary response of a trait to natural selection relies upon two genetic parameters. The heritability $\left(h^{2}\right)$ of the trait: i.e., the amount of phenotypic variation that can be attributed to genetic factors, and its genetic correlation with other fitness-related aspects of the phenotype: the degree to which the trait shares genetic variation with other characters under selection (Queller 2017). In this study, we used 8 years of corticosterone data from a wild population of barn owl nestlings to estimate the heritability of baseline and stress-induced corticosterone concentration, as well as the genetic correlation between these two traits, in order to shed light on the evolutionary potential of corticosterone levels. We show that baseline levels and stress-induced corticosterone response are both moderately heritable in our population of barn owl nestlings, within the range of what was observed in two previous studies in wild bird populations (Jenkins et al. 2014; Stedman et al. 2017). In contrast

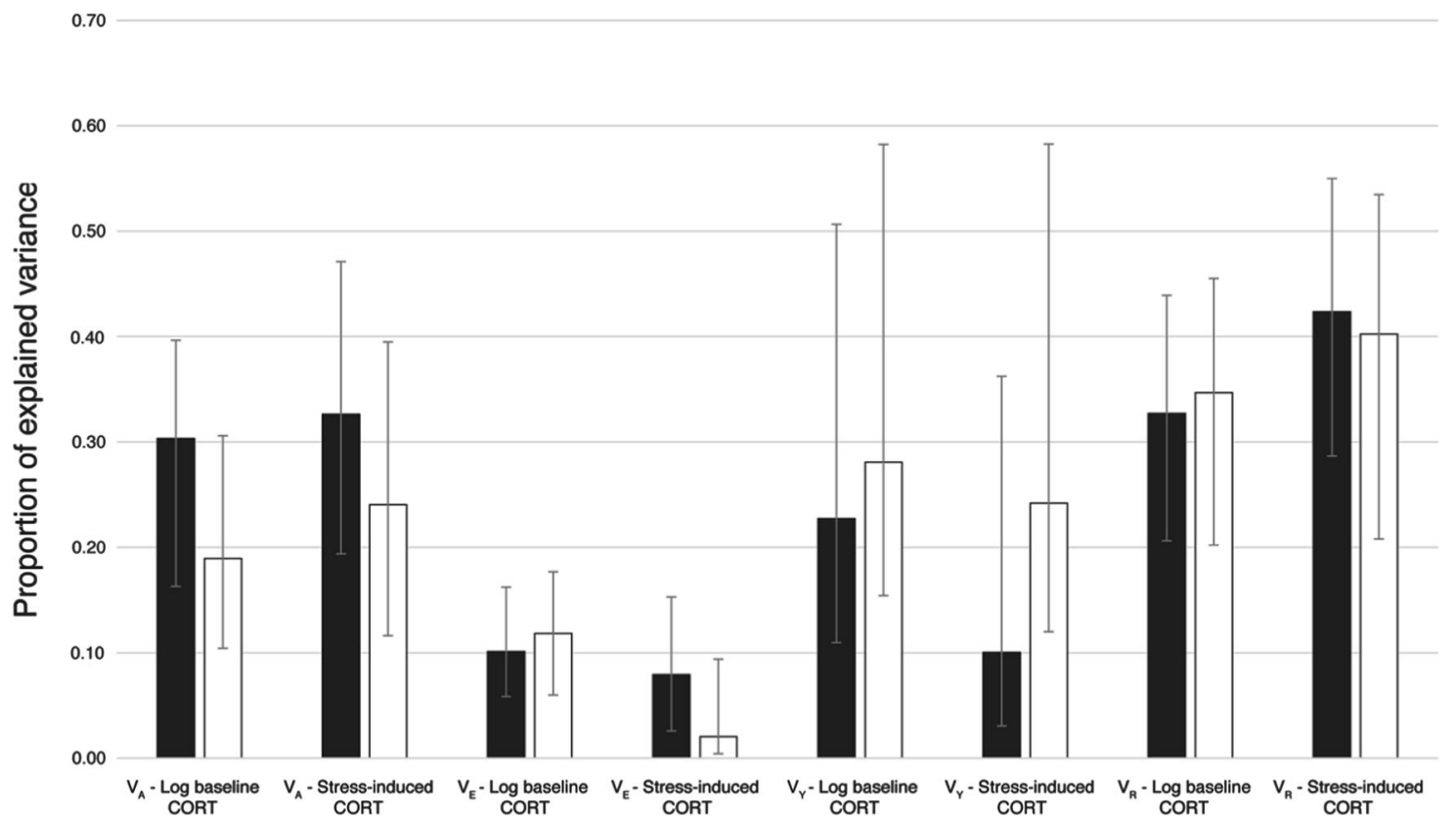

Fig. 1 Partition of total phenotypic variance in baseline and stressinduced corticosterone levels into additive genetic $\left(V_{\mathrm{A}}\right)$, common rearing environmental $\left(V_{\mathrm{E}}\right)$, year $\left(V_{\mathrm{Y}}\right)$ and residual variance $\left(V_{\mathrm{R}}\right)$, as estimated from a model without or with covariates. The additive genetic variance for corticosterone (CORT) was estimated from data on nestlings sampled between 6 and 67 days old using animal models without (black bars) or with covariates (white bars) influencing CORT levels (e.g., date and hour of sampling, sex and mass of nestlings, see Table S2). The error bars represent the $95 \%$ Bayesian credible intervals 
Table 2 Posterior estimation (with $95 \%$ Bayesian credible interval, $\mathrm{BCrI}$ ) of variance components for baseline and stress-induced corticosterone levels based on best models (see Table 1) without or with covariates

\begin{tabular}{lll}
\hline & $\begin{array}{l}\text { Baseline corticosterone } \\
(95 \% \mathrm{BCrI})\end{array}$ & $\begin{array}{l}\text { Stress-induced corticosterone } \\
(95 \% \mathrm{BCrI})\end{array}$ \\
\hline $\begin{array}{l}\text { Model 1 without covariates } \\
\text { Additive genetic variance } \\
\left(V_{\mathrm{A}}\right)\end{array}$ & $0.23(0.16-0.31)$ & $9.26(5.84-13.73)$ \\
Year $\left(V_{\mathrm{Y}}\right)$ & $0.19(0.06-0.60)$ & $2.54(0.69-13.54)$ \\
Rearing environment $\left(V_{\mathrm{E}}\right)$ & $0.08(0.06-0.13)$ & $2.67(0.79-4.34)$ \\
Residual variance $\left(V_{\mathrm{R}}\right)$ & $0.27(0.22-0.32)$ & $12.47(9.88-14.74)$ \\
Total variance $\left(V_{\mathrm{P}}\right)$ & $0.81(0.64-1.21)$ & $28.12(23.69-38.37)$ \\
Model 1 including covariates & & $6.89(3.93-10.65)$ \\
Additive genetic variance & $0.15(0.10-0.22)$ & $6.08(2.00-24.57)$ \\
$\left(V_{\mathrm{A}}\right)$ & $0.18(0.07-0.65)$ & $1.03(0.14-2.49)$ \\
Year $\left(V_{\mathrm{Y}}\right)$ & $0.09(0.06-0.13)$ & $10.47(8.62-12.75)$ \\
Rearing environment $\left(V_{\mathrm{E}}\right)$ & $0.26(0.21-0.30)$ & $26.01(20.09-43.19)$ \\
Residual variance $\left(V_{\mathrm{R}}\right)$ & $0.68(0.57-1.17)$ &
\end{tabular}

Note: Baseline and stress-induced corticosterone estimates are based on a logarithmic and a power of 0.7 transformation of corticosterone levels, respectively to previous studies that found no genetic correlation, we provide the first evidence for a strong positive genetic correlation between baseline and stress-induced response levels, indicating that, at least in our population, basal and stress-induced corticosterone levels might not evolve independently from each other.

Accounting for the effect of covariates known to be associated with corticosterone levels in the animal model (Supplementary Table S2), yielded qualitatively similar results, although some remarkable differences existed (Fig. 1). Regardless of whether covariates were added to the animal models or not, the models, including additive genetic (co)variance terms, were the most supported. Thus, evidence for an additive genetic (co)variance greater than zero in corticosterone levels is unlikely to be artefactual due to variation introduced to sampling nestlings from the same broods for instance. Although consistently smaller, the estimates obtained from the model with covariates were still within the same range of magnitude, like those obtained from the model without covariates (Fig. 1). Given this observation, the conclusions that can be derived from the models with or without covariates are the same: baseline levels and stress-induced corticosterone response show moderate heritability and are strongly genetically correlated in this data set.

To include fixed terms in animal models often leads to higher estimates of heritability, given that this normally reduces the magnitude of variance left unexplained by the model ( $V_{\mathrm{R}}$; Wilson 2008). However, in some circumstances, the inclusion of fixed effects can have the opposite effect: a decrease in heritability estimates, as observed in our study. Such a decrease in heritability estimates suggests that one or several covariates are somehow associated with the degree of relatedness among individuals (Wilson 2008). This can be due to nonbiological (e.g., sampling of related individuals under similar conditions), as well as biological factors (e.g., a genetic correlation between the trait and the covariate/s), rendering it difficult to decide whether the inclusion of fixed factors results in more or less accurate quantitative genetic estimates (Wilson 2008). In our case, we can expect both nonbiological and biological factors to account for the smaller estimates obtained when including covariates. On the one hand, there may exist genetic correlations between corticosterone levels and some of the included covariates (e.g., body condition: Kitaysky et al. 2001; Sockman and Schwabl 2001; Tilgar et al. 2017, see Table S2). On the other hand, we measured related full broods under similar conditions (same time of the day, date and current brood size), and this may have partly affected the animal model estimates, given that the type of family links that were more often represented in our pedigree were those among full siblings (see the Methods section, Pedigree: Kruuk and Hadfield 2007). Thus, the estimates from the models with or without fixed effects should be considered together (in addition to their credible intervals) as the range of heritability and genetic correlation of baseline and stress-induced corticosterone levels in our population.

Overall, the moderate heritability estimate for baseline and stress-induced corticosterone response suggests that an appreciable part of the variance in hormonal levels is genetically determined. This considerable amount of genetic variance may enable selective processes to mould the activity of the HPA axis and promote adaptation to specific environments (Abolins-Abols et al. 2016; Atwell et al. 2012; Fokidis et al. 2009; Müller et al. 2007; Partecke et al. 2006). Our analyses showed heritability estimates within 
the same range as those from other studies in wild bird populations. In our study, the animal model that included the fixed effect of different covariates produced similar heritability estimates for baseline $\left(h^{2}=0.19\right.$ [0.10-0.31]) and stress-induced corticosterone levels $\left(h^{2}=0.24\right.$ [0.12-0.40]) to those in a previous cross-fostering experiment made in barn swallows (baseline: $h^{2}=0.15$ [0.06-0.46], stress-induced: $h^{2}=0.34$ [0.13-0.6], Jenkins et al. 2014) and in a previous study conducted in tree swallows (baseline: $h^{2}=0.13$ [0.05-0.33], stress-induced: $h^{2}=0.18$ [0.08-0.42], Stedman et al. 2017). Our estimates were also within the range of heritability in corticosterone stress response observed in artificial selection experiments and in captivity (range of $h^{2}$ is 0.04-0.60; Brown and Nestor 1973; Cox et al. 2016; Evans et al. 2006; Odeh et al. 2003; Pottinger and Carrick 1999; Satterlee and Johnson 1988). Thus, the heritability of corticosterone estimates seems to be within the range of what has been estimated for other hormones (Coviello et al. 2010; Iserbyt et al. 2015; Pavitt et al. 2014; Travison et al. 2014). However, we believe that in order to generalise that baseline and stressinduced levels of corticosterone may often be moderately heritable, more studies are still required, particularly in wild populations.

Glucocorticoid hormones, such as corticosterone, are crucial in the mediation of physiological and behavioural processes and the ability of an organism to cope with environmental changes. Therefore, glucocorticoids are suggested to play an important role in the regulation of fitness traits, such as reproduction and survival of organisms. This is supported by the relationships found within different species between glucocorticoid levels and different fitness components (Angelier et al. 2009; Blas et al. 2007; Bonier et al. 2009b; Breuner et al. 2008; Goutte et al. 2010), including survival in adult barn owls (Béziers et al. 2019). In general, traits that are tightly associated with fitness, such as the HPA axis, are thought to have lower additive genetic variance, as alleles conferring a higher fitness are expected to be rapidly fixed by natural selection (Charmantier and Garant 2005; Kruuk et al. 2000; Roff 1997). Although this is the case for some fitness-related traits, a diversity of mechanisms, including fluctuating and disruptive selection, but also frequency-dependent selection and migration, are capable of maintaining genetic variance for traits, despite being closely associated with fitness (Bell 2010; Mousseau and Roff 1987). That the secretion of glucocorticoids can greatly vary across contexts and environments (Romero and Wingfield 2016), environmental fluctuations between years within our population could influence the fitness of different glucocorticoid phenotypes and maintain genetic variation. Interannual variation could limit the strength of selection if the optimal level of baseline corticosterone changes from one year to the next
(Vitousek et al. 2018). Further studies investigating the year-effect factors (e.g., food availability, weather conditions) on the regulation of corticosterone will be needed to understand better than the year-effect variation that we observed in our study, and how genetic variation in corticosterone levels is maintained.

The rearing environment explained less than $11 \%$ of the variance in baseline and stress-induced corticosterone levels (Fig. 1), which was less than the variance explained by the year effect. These estimates were lower than what was found in barn swallows $\left(V_{\mathrm{E}}>39 \%\right.$; Jenkins et al. 2014) and tree swallows $\left(V_{\mathrm{E}}>55 \%\right.$; Stedman et al. 2017). This indicates that environmental conditions experienced in a given year in different barn owl nests are more similar than environmental conditions experienced in different years. For instance, outbreaks of pathogens may occur in some specific years and affect all nests, and fluctuations in food supply may be more pronounced between years than between territories of barn owls. However, in barn swallows and tree swallows, the environmental conditions experienced between different colonies may have been more important and had major effects on corticosterone levels, contributing to the differences in corticosterone levels between colonies. These effects could be associated with the size of colonies (Møller 1987), promiscuity between nests (e.g., pathogens) or characteristics associated with the breeding site (e.g., food and predator abundance). In addition, we found that residual variance was much higher than the rearing environment, in particular for stress-induced corticosterone levels, suggesting that variation in corticosterone levels between siblings is influenced by additional factors, other than genetics or the environment of the nest. This might result from the experienced environment being very different between siblings because of the asynchronicity in the onset of incubation and hatching in barn owls. For instance, there can be a difference of more than 20 days between the first and the last hatchling. This is sufficient for the environmental and social conditions that each nestling experiences to change, and therefore for the HPA axis to be affected differently between siblings. Other factors, including maternal (Almasi et al. 2010; DuRant et al. 2010; Harris and Seckl 2011; Haussmann et al. 2012; Hayward and Wingfield 2004; Henriksen et al. 2011; Kapoor et al. 2006; Muneoka et al. 1997; Thayer et al. 2018; Uno et al. 1994; Weaver et al. 2004) and epigenetic effects during early development (Franklin et al. 2010; Lee et al. 2010; Nestler 2016), as well as parasite load or hatching asynchrony that can lead to competitive and developmental hierarchies (Evans 1996; Nilsson and Svensson 1996), can also alter the development or functioning of the endocrine system (Love et al. 2003; Schwabl 1999) and thus might potentially contribute to variation in corticosterone secretion between siblings, broods and years. 
A major difference in relation to previous studies in wild populations is that our study provides evidence for a positive genetic correlation between baseline and stress-induced corticosterone levels. From a proximate causation point of view, this strong correlation $(r=0.80)$ may be explained by the common synthetic pathway between baseline and stressinduced corticosterone, even if the baseline and stressresponse corticosterone actions are mediated by different receptors (Romero 2004). From an ultimate causation point of view, baseline corticosterone levels have been suggested to play a permissive action, permitting and priming the mechanisms by which an organism will respond to stress (Exton et al. 1972; Ingle 1952; Sapolsky et al. 2000; Seleznev Iu and Martynov 1982), which may result in selection for a genetic correlation between baseline and stress-induced levels. The existence of positive genetic correlations between baseline and stress-induced corticosterone levels is consistent with a previous study in great tits (Parus major), showing the interdependence between HPA axis measurements (i.e., baseline, stress-induced corticosterone levels and feedback capacities) and their correlation with personality (Baugh et al. 2017). However, the relationship between corticosterone levels and personality is not always clear, as in different populations, personality traits are found to correlate with change in stress-induced levels but not in baseline corticosterone levels (Baugh et al. 2012), whereas the contrary is found in other populations (Moyers et al. 2018). Moreover, studies selecting for high and low stress-induced corticosterone levels failed to find any correlated change in baseline corticosterone levels, further supporting the absence of a genetic correlation (Baugh et al. 2012; Cockrem et al. 2010; Pottinger and Carrick 1999; Satterlee and Johnson 1988). These studies, together with the studies of Jenkins et al. (2014) and Stedman et al. (2017) (although note that the smaller sample size of these two studies might have resulted in a lower power to detect a significant genetic correlation; Roff 1996; Wilson et al. 2010) suggest that the covariation between baseline and stress-induced corticosterone levels is probably not common to all species and populations. Further studies investigating the genetic covariation between baseline and stress-induced corticosterone levels in other species and populations are necessary to understand better why baseline and stress-induced corticosterone are genetically correlated in some species or populations but not in others. For instance, in some populations or species, a positive genetic correlation between baseline and stress-induced levels might have been selected against, owing to antagonistic selection on both traits. Genetic correlation can result from pleiotropy, where one or several loci regulate both traits, or by linkage disequilibrium, where alleles are tightly linked by nonrandom association. Disentangling the genetic mechanisms behind the secretion of corticosterone may also be necessary to understand the nature of the genetic covariation of baseline and stress-induced corticosterone levels within different species and populations.

In conclusion, this study sheds some light on the quantitative genetics of corticosterone levels, a rather understudied topic despite its relevance to understanding the evolution of stress response in vertebrates. It also emphasises the need to conduct similar studies on other species, in order to explain the general evolution of the glucocorticoid stress response and the HPA axis. However, future studies should also consider that the evolution of the HPA axis may be more complex, as the action of corticosterone not only depends on plasma corticosterone levels (Breuner et al. 2013), but also on the tissue-dependent density of receptors to which corticosterone binds (Seckl and Meaney 2004), the type of receptors (Landys et al. 2006; Romero 2004), the properties and concentration of binding proteins and enzymes involved in the secretion, transportation or degradation of corticosterone. Furthermore, like other endocrine traits, the HPA axis is a highly plastic trait that adjusts corticosterone levels according to environmental conditions and life-history stages. Therefore, the plasticity of the HPA axis is most likely to be the largest target of selection. Thus, determining the genetic basis of the plasticity of corticosterone levels will be an important step forward in comprehending the evolution of the HPA axis (Bonier and Martin 2016). Despite the fact that our study argues that baseline and stress-induced levels of corticosterone are strongly genetically linked in our population, selection may still be able to act independently on the signalling pathway of baseline activity and stress-induced response through all the factors depicted above. Additional studies may, therefore, investigate the dynamics between baseline and stress-induced corticosterone levels in more detail, and may also consider the level of genetic variation of other components involved in baseline and stress-response action in order to acknowledge better how phenotypic variation of the HPA axis may respond to the selection processes. Finally, due to the ubiquitous presence and pleiotropic effects of corticosterone, the HPA axis and corticosterone can potentially influence (and be influenced by) the evolution of many traits simultaneously. Therefore, determining the structure of the genetic covariance between hormone phenotypes, such as corticosterone, and the traits that they mediate, will provide a better comprehension of the evolutionary consequences of selection on the many hormone-mediated traits.

\section{Data archiving}

Data available from Dryad: https://doi.org/10.5061/dryad. hn0qm32. 
Acknowledgements We warmly thank all the people who helped us collect the field data from 1992 to 2016 . We are also grateful to the Swiss National Fond for having financed this study (grant $\mathrm{n}^{\circ} 3100 \mathrm{~A} 0-$ 104134 and $31003 \mathrm{~A}-127057$ to $\mathrm{LJ}$ and $\mathrm{n}^{\circ} 31003 \mathrm{~A}-120517$ to AR), V. Uva, D. Ferreira, and three anonymous reviewers for their comments and ideas that helped us improve the content of this paper. The corticosterone samples were taken under the legal authorisation of the "Service vétérinaire du canton de Vaud".

\section{Compliance with ethical standards}

Conflict of interest The authors declare that they have no conflict of interest.

Publisher's note: Springer Nature remains neutral with regard to jurisdictional claims in published maps and institutional affiliations.

\section{References}

Abolins-Abols M, Hope SF, Ketterson ED (2016) Effect of acute stressor on reproductive behavior differs between urban and rural birds. Ecol Evol 6:6546-6555

Albert FW, Shchepina O, Winter C, Rompler H, Teupser D, Palme R et al. (2008) Phenotypic differences in behavior, physiology and neurochemistry between rats selected for tameness and for defensive aggression towards humans. Horm Behav 53:413-421

Almasi B, Béziers P, Roulin A, Jenni L (2015) Agricultural land use and human presence around breeding sites increase stresshormone levels and decrease body mass in barn owl nestlings. Oecologia 179:89-101

Almasi B, Jenni L, Jenni-Eiermann S, Roulin A (2010) Regulation of stress response is heritable and functionally linked to melaninbased coloration. J Evol Biol 23:987-996

Almasi B, Roulin A, Jenni-Eiermann S, Breuner CW, Jenni L (2009) Regulation of free corticosterone and CBG capacity under different environmental conditions in altricial nestlings. Gen Comp Endocr 164:117-124

Almasi B, Roulin A, Korner-Nievergelt F, Jenni-Eiermann S, Jenni L (2012) Coloration signals the ability to cope with elevated stress hormones: effects of corticosterone on growth of barn owls are associated with melanism. J Evol Biol 25:1189-1199

Angelier F, Holberton RL, Marra PP (2009) Does stress response predict return rate in a migratory bird species? A study of American redstarts and their non-breeding habitat. P R Soc B 276:3545-3551

Atwell JW, Cardoso GC, Whittaker DJ, Campbell-Nelson S, Robertson KW, Ketterson ED (2012) Boldness behavior and stress physiology in a novel urban environment suggest rapid correlated evolutionary adaptation. Behav Ecol 23:960-969

Balloux F, Amos W, Coulson T (2004) Does heterozygosity estimate inbreeding in real populations? Mol Ecol 13:3021-3031

Baugh AT, Schaper SV, Hau M, Cockrem JF, de Goede P, van Oers K (2012) Corticosterone responses differ between lines of great tits (Parus major) selected for divergent personalities. Gen Comp Endocr 175:488-494

Baugh AT, Senft RA, Firke M, Lauder A, Schroeder J, Meddle SL et al. (2017) Risk-averse personalities have a systemically potentiated neuroendocrine stress axis: a multilevel experiment in Parus major. Horm Behav 93:99-108

Bell G (2010) Fluctuating selection: the perpetual renewal of adaptation in variable environments. Philos T R Soc B 365:87-97

Béziers P, Korner-Nievergelt F, Jenni L, Roulin A, Almasi B (2019). Survival of adult barn owls is linked to corticosterone levels. bioRxiv 101101/517201.
Béziers P, Roulin A (2016) Double brooding and offspring desertion in the barn owl Tyto alba. J Avian Biol 47:235-244

Blas J, Bortolotti GR, Tella JL, Baos R, Marchant TA (2007) Stress response during development predicts fitness in a wild, long lived vertebrate. Proc Natl Acad Sci USA 104:8880-8884

Bonier F, Martin PR (2016) How can we estimate natural selection on endocrine traits? Lessons from evolutionary biology. P R Soc B 283:20161887

Bonier F, Martin PR, Moore IT, Wingfield JC (2009a) Do baseline glucocorticoids predict fitness? Trends Ecol Evol 24:634-642

Bonier F, Moore IT, Martin PR, Robertson RJ (2009b) The relationship between fitness and baseline glucocorticoids in a passerine bird. Gen Comp Endocr 163:208-213

Bramanti P, White B, Luo S, Murray K (1997) Endocrine-exocrine modification procedures. Charles River Laboratories Reference Paper 10, p 3

Breuner CW, Delehanty B, Boonstra R (2013) Evaluating stress in natural populations of vertebrates: total CORT is not good enough. Funct Ecol 27:24-36

Breuner CW, Patterson SH, Hahn TP (2008) In search of relationships between the acute adrenocortical response and fitness. Gen Comp Endocr 157:288-295

Brown KI, Nestor KE (1973) Some physiological responses of Turkeys selected for high and low adrenal response to cold stress. Poult Sci 52:1948-1954

Buckley TM, Schatzberg AF (2005) Review: on the interactions of the hypothalamic-pituitary-adrenal (HPA) axis and sleep: normal HPA axis activity and circadian rhythm, exemplary sleep disorders. J Clin Endocr Metab 90:3106-3114

Carere C, Groothuis TGG, Mostl E, Daan S, Koolhaas JM (2003) Fecal corticosteroids in a territorial bird selected for different personalities: daily rhythm and the response to social stress. Horm Behav 43:540-548

Carsia RV, Harvey A (2000) Adrenals. In: Causey GW (ed) Sturkie's avian physiology, 5th edn. Academic Press, London, p 489-522

Charmantier A, Garant D (2005) Environmental quality and evolutionary potential: lessons from wild populations. P R Soc B 272:1415-1425

Charmantier A, Reale D (2005) How do misassigned paternities affect the estimation of heritability in the wild? Mol Ecol 14:2839-2850

Chennaoui M, Merino DG, Lesage J, Drogou C, Guezennec CY (2002) Effects of moderate and intensive training on the hypothalamo-pituitary-adrenal axis in rats. Acta Physiol Scand 175:113-121

Cockrem JF, Candy EJ, Castille SA, Satterlee DG (2010) Plasma corticosterone responses to handling in Japanese quail selected for low or high plasma corticosterone responses to brief restraint. Brit Poult Sci 51:453-459

Coviello AD, Zhuang WV, Lunetta KL, Ulloor J, Zhang A, Karasik D et al. (2010) Heritability of circulating testosterone and SHBG in adult women from the Framingham heart study. Endocr Rev 31: S41-S41

Cox RM, McGlothlin JW, Bonier F (2016) Hormones as mediators of phenotypic and genetic integration: an evolutionary genetics approach. Integr Comp Biol 56:126-137

Crespi EJ, Williams TD, Jessop TS, Delehanty B (2013) Life history and the ecology of stress: how do glucocorticoid hormones influence life-history variation in animals? Funct Ecol 27:93-106

Dallman MF, Strack AM, Akana SF, Bradbury MJ, Hanson ES, Scribner KA et al. (1993) Feast and famine-critical role of glucocorticoids with insulin in daily energy-flow. Front Neuroendocr 14:303-347

Ducret V, Gaigher A, Simon C, Goudet J, Roulin A (2016) Sexspecific allelic transmission bias suggests sexual conflict at MC1R. Mol Ecol 25:4551-4563 
DuRant SE, Hepp GR, Moore IT, Hopkins BC, Hopkins WA (2010) Slight differences in incubation temperature affect early growth and stress endocrinology of wood duck (Aix sponsa) ducklings. J Exp Biol 213:45-51

Evans MR, Roberts ML, Buchanan KL, Goldsmith AR (2006) Heritability of corticosterone response and changes in life history traits during selection in the zebra finch. J Evol Biol 19:343-352

Evans RM (1996) Hatching asynchrony and survival of insurance offspring in an obligate brood reducing species, the American white pelican. Behav Ecol Sociobiol 39:203-209

Exton JH, Friedmann N, Wong EHA, Brineaux JP, Corbin JD, Park CR (1972) Interaction of glucocorticoids with glucagon and epinephrine in control of gluconeogenesis and glycogenolysis in liver and of lipolysis in adipose-tissue. J Biol Chem 247:3579

Fairbanks LA, Jorgensen MJ, Bailey JN, Breidenthal SE, Grzywa R, Laudenslager ML (2011) Heritability and genetic correlation of hair cortisol in vervet monkeys in low and higher stress environments. Psychoneuroendocrino 36:1201-1208

Fevolden SE, Roed KH, Fjalestad KT, Stien J (1999) Poststress levels of lysozyme and cortisol in adult rainbow trout: heritabilities and genetic correlations. J Fish Biol 54:900-910

Flatt T, Tu MP, Tatar M (2005) Hormonal pleiotropy and the juvenile hormone regulation of Drosophila development and life history. Bioessays 27:999-1010

Fokidis HB, Orchinik M, Deviche P (2009) Corticosterone and corticosteroid binding globulin in birds: relation to urbanization in a desert city. Gen Comp Endocr 160:259-270

Franklin TB, Russig H, Weiss IC, Graff J, Linder N, Michalon A et al. (2010) Epigenetic transmission of the impact of early stress across generations. Biol Psychiat 68:408-415

Garland T, Saltzman W, Garland T (2016) Hormones and the evolution of complex traits: insights from artificial selection on behavior. Integr Comp Biol 56:E73-E73

Goutte A, Angelier F, Welcker J, Moe B, Clement-Chastel C, Gabrielsen GW et al. (2010) Long-term survival effect of corticosterone manipulation in Black-legged kittiwakes. Gen Comp Endocr 167:246-251

Hadfield JD (2010) MCMC methods for multi-response generalized linear mixed models: the MCMCglmm R package. J Stat Softw 33:1-22

Harris A, Seckl J (2011) Glucocorticoids, prenatal stress and the programming of disease. Horm Behav 59:279-289

Harvey S, Hall TR (1990) Hormones and stress in birds-activation of the hypothalamo-pituitary-adrenal axis. Progress Comp Endocrinol 342:453-460

Hau M, Goymann W (2015) Endocrine mechanisms, behavioral phenotypes and plasticity: Known relationships and open questions. Front Zool 12:S7

Haussmann MF, Longenecker AS, Marchetto NM, Juliano SA, Bowden RM (2012) Embryonic exposure to corticosterone modifies the juvenile stress response, oxidative stress and telomere length. P R Soc B 279:1447-1456

Hayward LS, Wingfield JC (2004) Maternal corticosterone is transferred to avian yolk and may alter offspring growth and adult phenotype. Gen Comp Endocr 135:365-371

Henriksen R, Rettenbacher S, Groothuis TGG (2011) Prenatal stress in birds: pathways, effects, function and perspectives. Neurosci Biobehav R 35:1484-1501

Henry I, Antoniazza S, Dubey S, Simon C, Waldvogel C, Burri R et al (2013) Multiple paternity in polyandrous barn owls (Tyto alba). Plos One 8:e80112

Huey RB, Rosenzweig F (2009) Laboratory evolution meets Catch-22 balancing simplicity and realism. University of California Press, Berkeley

Ingle DJ (1952) The role of the adrenal cortex in homeostasis. J Endocrinol 8:23-37
Iserbyt A, Eens M, Müller W (2015) Sexually antagonistic selection during parental care is not generated by a testosterone-related intralocus sexual conflict-insights from full-sib comparisons. Sci Rep-Uk 5:17715

Jenkins BR, Vitousek MN, Hubbard JK, Safran RJ (2014) An experimental analysis of the heritability of variation in glucocorticoid concentrations in a wild avian population. P R Soc B 281:20141302

Kadarmideen HN, Janss LLG (2007) Population and systems genetics analyses of cortisol in pigs divergently selected for stress. Physiol Genom 29:57-65

Kapoor A, Dunn E, Kostaki A, Andrews MH, Matthews SG (2006) Fetal programming of hypothalamo-pituitary-adrenal function: prenatal stress and glucocorticoids. J Physiol-Lond 572:31-44

Kawashima H, Saito T, Yoshizato H, Fujikawa T, Sato Y, McEwen BS et al. (2004) Endurance treadmill training in rats alters $\mathrm{CRH}$ activity in the hypothalamic paraventricular nucleus at rest and during acute running according to its period. Life Sci 76:763-774

Kempenaers B, Peters A, Foerster K (2008) Sources of individual variation in plasma testosterone levels. Philos $\mathrm{T} R$ Soc $\mathrm{B}$ 363:1711-1723

King BM (1988) Glucocorticoids and hypothalamic obesity. Neurosci Biobehav R 12:29-37

Kitaysky AS, Kitaiskaia EV, Wingfield JC, Piatt JF (2001) Dietary restriction causes chronic elevation of corticosterone and enhances stress response in red-legged kittiwake chicks. J Comp Physiol B 171:701-709

Kruuk LE, Clutton-Brock TH, Slate J, Pemberton JM, Brotherstone S, Guinness FE (2000) Heritability of fitness in a wild mammal population. Proc Natl Acad Sci USA 97:698-703

Kruuk LEB, Hadfield JD (2007) How to separate genetic and environmental causes of similarity between relatives. J Evol Biol 20:1890-1903

Kuo T, McQueen A, Chen TC, Wang JC (2015) Regulation of glucose homeostasis by glucocorticoids. In: Wang JC, Harris C (eds) Vol. 872, Glucocorticoid signaling: from molecules to mice to man. Springer-Verlag Berlin, Berlin, p 99-126

Landys MM, Ramenofsky M, Wingfield JC (2006) Actions of glucocorticoids at a seasonal baseline as compared to stress-related levels in the regulation of periodic life processes. Gen Comp Endocr 148:132-149

Lee RS, Tamashiro KLK, Yang XJ, Purcell RH, Harvey A, Willour VL et al. (2010) Chronic corticosterone exposure increases expression and decreases deoxyribonucleic acid methylation of Fkbp5 in mice. Endocrinology 151:4332-4343

Love OP, Bird DM, Shutt LJ (2003) Plasma corticosterone in American kestrel siblings: effects of age, hatching order, and hatching asynchrony. Horm Behav 43:480-488

Mills SC, Koskela E, Mappes T (2012) Intralocus sexual conflict for fitness: sexually antagonistic alleles for testosterone. P R Soc B 279:1889-1895

Møller AP (1987) Advantages and disadvantages of coloniality in the swallow, Hirundo-Rustica. Anim Behav 35:819-832

Mousseau TA, Roff DA (1987) Natural-selection and the heritability of fitness components. Heredity 59:181-197

Moyers SC, Adelman JS, Farine DR, Moore IT, Hawley DM (2018) Exploratory behavior is linked to stress physiology and social network centrality in free-living house finches (Haemorhous mexicanus). Horm Behav 102:105-113

Müller C, Almasi B, Roulin A, Breuner CW, Jenni-Eiermann S, Jenni L (2009) Effects of corticosterone pellets on baseline and stressinduced corticosterone and corticosteroid-binding-globulin. Gen Comp Endocr 160:59-66

Müller C, Jenni-Eiermann S, Blondel J, Perret P, Caro SP, Lambrechts $M$ et al. (2006) Effect of human presence and handling on 
circulating corticosterone levels in breeding blue tits (Parus caeruleus). Gen Comp Endocr 148:163-171

Müller C, Jenni-Eiermann S, Blondel J, Perret P, Caro SP, Lambrechts MM et al. (2007) Circulating corticosterone levels in breeding blue tits Parus caeruleus differ between island and mainland populations and between habitats. Gen Comp Endocr 154:128-136

Muneoka K, Mikuni M, Ogawa T, Kitera K, Kamei K, Takigawa M et al. (1997) Prenatal dexamethasone exposure alters brain monoamine metabolism and adrenocortical response in rat offspring. Am J Physiol 273(5 Pt 2):R1669-R1675

Nestler EJ (2016) Transgenerational epigenetic contributions to stress responses: fact or fiction? Plos Biol 14:e1002486

Nilsson JA, Svensson M (1996) Sibling competition affects nestling growth strategies in marsh tits. J Anim Ecol 65:825-836

Odeh FM, Cadd GG, Satterlee DG (2003) Genetic characterization of stress responsiveness in Japanese quail. 2. Analyses of maternal effects, additive sex linkage effects, heterosis, and heritability by diallel crosses. Poult Sci 82:31-35

Partecke J, Schwabl I, Gwinner E (2006) Stress and the city: urbanization and its effects on the stress physiology in European blackbirds. Ecology 87:1945-1952

Pavitt AT, Walling CA, Pemberton JM, Kruuk LEB (2014) Heritability and cross-sex genetic correlations of early-life circulating testosterone levels in a wild mammal. Biol Lett 10:1-4

Pemberton JM (2008) Wild pedigrees: the way forward. P R Soc B 275:613-621

Pottinger TG, Carrick TR (1999) Modification of the plasma cortisol response to stress in rainbow trout by selective breeding. Gen Comp Endocrinol 116:122-132

Py I, Ducrest AL, Duvoisin N, Fumagalli L, Roulin A (2006) Ultraviolet reflectance in a melanin-based plumage trait is heritable. Evol Ecol Res 8:483-491

Queller DC (2017) Fundamental theorems of evolution. Am Nat 189:345-353

Rodrigues SM, LeDoux JE, Sapolsky RM (2009) The influence of stress hormones on fear circuitry. Annu Rev Neurosci 32:289-313

Roff DA (1996) The evolution of genetic correlations: an analysis of patterns. Evolution 50:1392-1403

Roff DA (1997) Evolutionary quantitative genetics. Chapman and Hall, New York

Romero LM (2004) Physiological stress in ecology: lessons from biomedical research. Trends Ecol Evol 19:249-255

Romero LM, Reed JM (2005) Collecting baseline corticosterone samples in the field: is under 3 min good enough? Comp Biochem Phys A 140:73-79

Romero LM, Wingfield JC (2016) Tempests, poxes, predators, and people: stress in wild animals and how to cope. Oxford University Press: Oxford

Roulin A, Almasi B, Jenni L (2010) Temporal variation in glucocorticoid levels during the resting phase is associated in opposite way with maternal and paternal melanic coloration. J Evol Biol 23:2046-2053

Roulin A, Almasi B, Rossi-Pedruzzi A, Ducrest AL, Wakamatsu K, Miksik I et al. (2008) Corticosterone mediates the conditiondependent component of melanin-based coloration. Anim Behav 75:1351-1358

San-Jose LM, Ducret V, Ducrest AL, Simon C, Roulin A (2017) Beyond mean allelic effects: a locus at the major color gene MC1R associates also with differing levels of phenotypic and genetic (co)variance for coloration in barn owls. Evolution 71:2469-2483

Sapolsky RM, Romero LM, Munck AU (2000) How do glucocorticoids influence stress responses? Integrating permissive, suppressive, stimulatory, and preparative actions. Endocr Rev 21:55-89

Satterlee DG, Johnson WA (1988) Selection of Japanese quail for contrasting blood corticosterone response to immobilization. Poult Sci 67:25-32

Schwabl H (1999) Developmental changes and among-sibling variation of corticosterone levels in an altricial avian species. Gen Comp Endocr 116:403-408

Seckl JR, Meaney MJ (2004) Glucocorticoid programming. Ann NY Acad Sci 1032:63-84

Seleznev Iu M, Martynov AV (1982) Permissive effect of glucocorticoids in catecholamine action in the heart: possible mechanism. J Mol Cell Cardiol 14(Suppl 3):49-58

Sgro CM, Hoffmann AA (2004) Genetic correlations, tradeoffs and environmental variation. Heredity 93:241-248

Sockman KW, Schwabl H (2001) Plasma corticosterone in nestling American kestrels: effects of age, handling stress, yolk androgens, and body condition. Gen Comp Endocr 122:205-212

Stedman JM, Hallinger KK, Winkler DW, Vitousek MN (2017) Heritable variation in circulating glucocorticoids and endocrine flexibility in a free-living songbird. J Evol Biol 30:1724-1735

Stowe M, Rosivall B, Drent PJ, Mostl E (2010) Selection for fast and slow exploration affects baseline and stress-induced corticosterone excretion in Great tit nestlings, Parus major. Horm Behav $58: 864-871$

Thayer ZM, Wilson MA, Kim AW, Jaeggi AV (2018) Impact of prenatal stress on offspring glucocorticoid levels: a phylogenetic meta-analysis across 14 vertebrate species. Sci Rep-Uk 8:9

Tilgar V, Lind M, Lodjak J, Moks K (2017) Corticosterone response as an age-specific mediator of nestling body mass in a wild Passerine. Physiol Biochem Zool 90:512-521

Travison TG, Zhuang WV, Lunetta KL, Karasik D, Bhasin S, Kiel DP et al. (2014) The heritability of circulating testosterone, oestradiol, oestrone and sex hormone binding globulin concentrations in men: the Framingham Heart Study. Clin Endocrinol 80:277-282

Uno H, Eisele S, Sakai A, Shelton S, Baker E, DeJesus O et al. (1994) Neurotoxicity of glucocorticoids in the primate brain. Horm Behav 28:336-348

Vitousek MN, Taff CC, Hallinger KK, Zimmer C, Winkler AM (2018) Hormones and fitness: Evidence for trade-offs in glucocorticoid regulation across contexts. Front Ecol Evol 6:42

Weaver ICG, Cervoni N, Champagne FA, D'Alessio AC, Sharma S Jr $S$ et al. (2004) Epigenetic programming by maternal behavior. Nat Neurosci 7:847-854

Wilson AJ (2008) Why h(2) does not always equal V-A/V-P? J Evol Biol 21:647-650

Wilson AJ, Reale D, Clements MN, Morrissey MM, Postma E, Walling CA et al. (2010) An ecologist's guide to the animal model. J Anim Ecol 79:13-26

Wingfield JC, Maney DL, Breuner CW, Jacobs JD, Lynn S, Ramenofsky M et al. (1998) Ecological bases of hormone-behavior interactions: the "emergency life history stage". Am Zool 38:191-206

Zera AJ, Harshman LG, Williams TD (2007) Evolutionary endocrinology: the developing synthesis between endocrinology and evolutionary genetics. Annu Rev Ecol Evol S 38:793-817 